Missed Diagnosis

\title{
Acute presentation of cystic fibrosis in an adult
}

\author{
K.R. Gardiner and B. Cranley \\ Department of Surgery, Daisy Hill Hospital, Newry BT35 8DR, UK.
}

\begin{abstract}
Summary: The case history of a female patient presenting at the age of 25 with cystic fibrosis is described. Despite longstanding significant respiratory symptoms, she did not attend hospital until she was 23 and then failed to appear for further investigations and treatment.

At the age of 25, she was admitted with a severe lower respiratory tract infection and respiratory failure. Within 5 days, acute intestinal obstruction developed which was unresponsive to conservative therapy. At laparotomy the small bowel was obstructed just proximally to the ileocaecal valve by solid faecal material. Post-operatively, the diagnosis of cystic fibrosis was confirmed by quantitative pilocarpine iontophoresis tests.

The literature is reviewed regarding the adult presentations of cystic fibrosis and the recommended treatment for distal intestinal obstruction syndrome. The presence of adult cystic patients within the community is emphasized.
\end{abstract}

\section{Introduction}

Cystic fibrosis is the most common autosomal recessive disorder in white populations with a prevalence of approximately one in two thousand. Hellerstein ${ }^{1}$ reported the first case of cystic fibrosis to be newly diagnosed over the age of 20 . Subsequent case reports of cystic fibrosis diagnosed in adulthood are rare. ${ }^{2} \mathrm{We}$ report an acute presentation of cystic fibrosis in an adult who developed complete distal small bowel obstruction within 5 days of admission with a chest infection.

\section{Case report}

A 25 year old female presented with a 3-day history of increasing cough, green sputum, and dyspnoea. There was a history of cough since birth with later development of tenacious yellow-green sputum, and shortness of breath on exertion. There was no bowel disturbance or steatorrhoea, but she had lost $12 \mathrm{~kg}$ in weight in 2 years. She had taken part in physical education at school and attended 'discos'. The only previous hospital attendance was at an outpatient clinic some 18 months previously when she was working as a machinist in a nylon factory. At that time she complained of recurring chest infections. She was

Correspondence: K.R. Gardiner M.B., F.R.C.S., Department of Surgery, Craigavon Area Hospital, Craigavon, Co. Armagh BT63 5QQ, UK.

Accepted: 30 November 1988 cyanosed and had coarse crepitations on auscultation of her chest. She was felt to have bronchiectasis but failed to attend for sweat test or follow-up.

On examination, she was dyspnoeic at rest with central cyanosis and finger clubbing. She had pectus carinatum with bilaterally poor expansion, coarse crepitations and a wheeze. Investigations revealed a leucocyte count of $37.4 \times 10^{9}$ cells/litre, and $\mathrm{PO}_{2}$ $4.8 \mathrm{kPa}, \mathrm{PCO}_{2} 7.4 \mathrm{kPa}$, and $\mathrm{pH} 7.28$ on room air. Chest radiography demonstrated over-inflation of the lungs with widespread bronchial wall thickening and numerous small patches of consolidation throughout both lung fields. Electrocardiography showed a sinus tachycardia, right axis deviation and right atrial enlargement. Klebsiella and Pseudomonas species were cultured from the sputum. She was treated with oxygen and salbutamol by mask, intravenous aminophylline, hydrocortisone and antibiotics.

Five days after admission she complained of crampy abdominal pain, vomiting and constipation. There was generalized abdominal distension, tenderness and increased bowel sounds. Erect and supine abdominal radiographs revealed multiple small bowel air-fluid levels with moderately dilated loops.

She was treated conservatively with nasogastric suction and intravenous rehydration, but failed to improve. At laparotomy the terminal ileum was obstructed by solid faecal material over a length of $30 \mathrm{~cm}$. An enterotomy was made just distal to the obstruction, and the faecal material 'milked' out. The enterotomy was then exteriorized. Post-operatively

(C) The Fellowship of Postgraduate Medicine, 1989 
she required ventilation for 4 days and a considerable period of convalescence. During this period, the diagnosis of cystic fibrosis was confirmed by quantitative pilocarpine iontophoresis tests.

\section{Discussion}

Cystic fibrosis is a multi-system disorder involving all exocrine glands. ${ }^{3}$ With vigorous physiotherapy and antibiotic therapy the outlook has changed with up to $75 \%$ of affected individuals now reaching adult life. ${ }^{2}$ In addition, up to $5 \%$ of patients with cystic fibrosis are not diagnosed until adulthood. ${ }^{4}$ This may reflect a mild end of a continuous spectrum of clinical disease or an improved prognosis in those without pancreatic insufficiency. ${ }^{2}$ Others may have been incorrectly diagnosed as suffering from coeliac disease, tuberculosis, bronchiectasis or bronchial asthma. ${ }^{5}$ The diagnosis of cystic fibrosis may be difficult in adults as they often do not present with the classic textbook description of the disease.

The commonest presentation in adults is recurrent chest infections. ${ }^{3}$ Pancreatic insufficiency, nasal polyps, and paranasal sinusitis, and infertility are other common manifestations. Intestinal complica-

\section{References}

1. Hellerstein, H.K. Cystic fibrosis of the pancreas in an adult. Ohio Med J 1946, 42: 616-617.

2. Hunt, B. \& Geddes, D.M. Newly diagnosed cystic fibrosis in middle and later life. Thorax 1985, 40: 23-26.

3. Godbey, M.W. Cystic fibrosis in adults. J Mississippi State Med Assoc 1985, 26: 161-163.

4. Davis, P.B. Cystic fibrosis in adults. In: Lloyd-Still, J.D. (ed) Textbook of Cystic Fibrosis. John Wright-PSG, London, 1983, pp. 351-369. tions are also frequent in established cases with $32 \%$ की patients experiencing at least one episode of constipan tion and $9 \%$ developing distal intestinal obstruction syndrome. ${ }^{6}$ Precipitating events such as dehydratio associated with fever or pulmonary exacerbation. newly prescribed narcotic analgesics, discontinuation. of enzyme supplements or a change in the diet may be identified in the majority of patients with dista intestinal obstruction syndrome. ${ }^{6}$

Patients known to have cystic fibrosis with distas intestinal obstruction syndrome should be treate with rehydration, gastric decompression, enemas an nasogastric infusions of acetylcysteine, pancreatin or balanced intestinal lavage solution. ${ }^{6}$ If such measures are unsuccessful then operative intervention is neces $\overrightarrow{\vec{\omega}}$

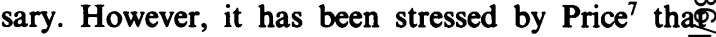
significant deterioration in lung function occurs afte even brief anaesthesia.

The condition of the chest in cystic fibrosis usuallig determines the length of survival. Early diagnosis in this case, with referral to a cystic fibrosis centre, mag have retarded the progressive pulmonary damage and prevented the distal intestinal obstruction syndromeThis case serves to emphasize the existence of undiag nosed patients suffering from cystic fibrosis within thecommunity.

5. Shwachman, H., Kowalski, M. \& Shaw K-T. Cy fibrosis: a new outlook. Medicine 1979, 56: 129-149:

6. Rubinstein, S., Moss, R \& Lewiston, N. Constipation an meconium ileus equivalent in patients with cystic fibrosiso Pediatrics 1986, 78: 473-479.

7. Price, J.F. The need to avoid general anaesthesia in cysti fibrosis. J R Soc Med 1986, 79 (Suppl 12): 10-12. 